\title{
Perturbed Collocation Method For Solving Singular Multi-order Fractional Differential Equations of Lane-Emden Type
}

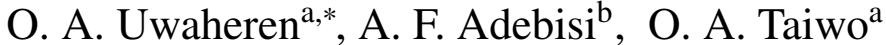 \\ ${ }^{a}$ Department of Mathematics, University of Ilorin, Ilorin, Nigeria \\ ${ }^{b}$ Department of Mathematics, Osun State University, Oshogbo, Nigeria
}

\begin{abstract}
In this work, a general class of multi-order fractional differential equations of Lane-Emden type is considered. Here, an assumed approximate solution is substituted into a slightly perturbed form of the general class and the resulting equation is collocated at equally spaced interior points to give a system of linear algebraic equations which are then solved by suitable computer software; Maple 18.
\end{abstract}

Keywords: Perturbed Collocation Method, Legendre polynomial, Singular multi-order differential equations, Lane-Emden equations

\section{Article History:}

Received: 17 March 2020

Received in revised form: 15 May 2020

Accepted for publication: 16 May 2020

Published: 01 August 2020

(c)2020 Journal of the Nigerian Society of Physical Sciences. All rights reserved. Communicated by: T. Latunde

\section{Introduction}

Perturbation Collocation Method is a technique of numerical approximation. It has been described as a very useful tool for solving differential and integro-differential equations of different kinds [1, 2, 3]. Fractional order differential equation is a special kind of differential equations with fractional order derivative say $\alpha$, which is a non-integer. Many phenomena in science and engineering resulting in fractional order differential equations have been successfully represented using mathematical models in the field of mechanics, elasticity, science, education to mention but a few. Most often, the equations arising from the mathematical modelling of fractional order differential equations are difficult to solve analytically. This is because many of them do not have solutions in closed form

\footnotetext{
${ }^{*}$ Corresponding author tel. no: $+23480 \times x \times x 572$

Email address: uwaheren. ao@unilorin.edu.ng (O. A. Uwaheren )
}

and hence seeking an approximate solution by numerical treatment becomes helpful. To achieve this, a lot of numerical methods have been advanced. [4, 5] proposed Variational Iteration Method (VIM) and used the method to solve multi-order fractional integro-differential equations and singular IVPs of LaneEmden type respectively. Their results showed that the method is an accurate one that yields the exact solution within a few iterations. Their study, however, noted that in some cases, the method may require more calculations which will add some difficulties. [6] solved nonlinear multi-order fractional differential equations using Legendre Pseudo-Spectral Method (LPSM). The results reveal that the method is effective as a small number of shifted Legendre polynomials were needed to obtain a satisfactory result. [7] presented a numerical solution method for solving fractional differential equations using Bernstein polynomials. Other methods used by many researchers are Chebyshev Wavelet method [8], Chebyshev Polynomial Method [9, 10] Collocation Method [11], Pade Approximate Method [12], 
etc. [12] described the Lane-Emden differential equation as a singular initial value problem relating to second-order differential equations which have been used to model several phenomena in mathematical physics and astrophysics.

According to [13], Lane-Emden type of differential equation is used to describe a variety of phenomena in physics and astrophysics, including isothermal gas spheres and thermionic currents and determining their numerical solutions is very challenging because of the singularity behaviours at the point of origin. The study also solved some fractional order differential equations of Lane-Emden type using the collocation method and admitted that a collocation method is a suitable tool for solving a class of Lane-Emden type of differential equations. [14] worked on the numerical solution of Lane-Emden type equation using multilayer perceptron neural network method. The study aimed at producing an optimal solution of LaneEmden equation with less computation using multilayer perceptron artificial neural network technique. The results obtained show that the technique can develop into an effective approach for solving Lane-Emden type problems with less computational time and memory space.

In this study, we are concerned with multi-order fractional differential equations of Lane-Emden type and their solutions by perturbation collocation method. To transform the problems to a system of linear algebraic equations, the collocation method algorithms as given by [13] was implemented.

Other researchers that have solved different problems of LaneEmden type using different methods include [15, 16, 17 ].

The general form of the singular multi-order fractional differential equation is given as

$$
\begin{aligned}
& \sum_{i=0}^{n} P_{i} y^{(i)}(x)+D^{\alpha}(y(x))+\frac{k}{x^{\alpha-\beta}} D^{\beta}(y(x)) \\
& +\frac{k}{x^{\alpha-2}}(y(x))=f(x)
\end{aligned}
$$

subject to conditions

$$
y(0)=A, \quad y^{\prime}(0)=B
$$

where $A, B$ are constants, $P_{i},(i=0,1,2, . . n)$ are arbitrary constants to be determined, $n$ is the highest integer order derivative, $\alpha$ and $\beta$ are fractional order derivatives, and $\alpha>\beta$.

\section{Definition of Relevant Terms}

\subsection{Assumed Approximate Solution}

An Assumed Approximate Solution also called Trial Solution refers to a predetermined polynomial taken to represent the solution of the problem been solved.

\subsection{Maple 18}

Is a mathematical software that is capable of Performing mathematical computation, for example, solving an equation symbolically or numerically, or construct Maple objects etc depending on the command.

\subsection{Fractional Differential Equation}

A differential equation is called a fractional differential equation if it contains at least one fractional order derivatives, $D^{\alpha}$ of the unknown function $y(x)$. The general form of a fractional differential equation is given as

$$
D^{\alpha} y(x)=f(x, y(x))
$$

subject to the conditions: $D^{\alpha} y(0)=\omega_{k}, \quad k=0,1, \ldots, n$ where $D^{\alpha}$ is the fractional order derivative in the Caputo sense and $\alpha$ is a non-integer value, $n=\lceil\alpha\rceil$, called the ceiling $\alpha, \alpha>0$ is the highest order of the equation.

The operator $D^{\alpha}$ is defined as

$$
D_{*}^{\alpha} f(x)=\frac{1}{\Gamma(m-\alpha)} \int_{0}^{x}(x-t)^{m-\alpha-1} \frac{d^{m}}{d t^{m}} f(t) d t
$$

in the Caputo sense for function $f(x)$ with $(m-1)$ absolute continuous derivatives and Riemann-liouville defined it as

$$
{ }_{c} D_{x}^{\beta} f(x)=\frac{1}{\Gamma(n-\beta)} \frac{d^{n}}{d x^{n}} \int_{c}^{x}(x-t)^{\beta-1} f(t) d t
$$

The advantage of the Caputo's fractional derivative is that one can specify the initial conditions of the fractional differential equation in classical form. i.e.

$$
D^{(k)} y(k)=b_{k}, \quad k=0,1,2, \cdots, m-1
$$

The generalized factorial form of non-integer order derivatives in Euler's Gamma function $f(x)=x^{m}$ is given as

$$
\frac{d^{\alpha}}{d x^{\alpha}} x^{m}=\frac{\Gamma(m+1)}{\Gamma(m-n+1)} x^{n-m}
$$

Equation (7) is a property of fractional derivatives given in Euler gamma functions which makes the definition more suitable and compatible for application.

\subsection{Multi-order Fractional differential Equations of Lane-Emden type}

A fractional multi-order differential equation of Lane-Emden type is of the form equation (1). However, when the highest order derivative of the equation is fractional, then equation (1) becomes:

$$
D^{\alpha}(y(t))+\frac{k}{t^{\alpha-\beta}} D^{\beta}(y(t))+\frac{k}{t^{\alpha-2}}(y(t))=f(t)
$$

subject to conditions

$$
y(0)=A, \quad y^{\prime}(0)=B
$$

where $A, B, \alpha$ and $\beta$ are as described earlier. 


\section{Methodology}

Consider a general class of multi-order fractional differential equation of Lane-Emden type given in equation (1) together with the conditions in equation (2).

In order to solve equations (1) and (2), an assumed approximate solution of the form:

$$
y_{N}(t)=\sum_{j=0}^{N} a_{j} L_{j}(t)
$$

is taken. Where $L_{j}(t)$ is Legendre polynomial and $N$ is the degree of the assumed approximant.

Thus equation(10) is substituted into a slightly perturbed equation (1) to give

$$
\begin{aligned}
& \sum_{i=0}^{n} P_{i}\left\{\sum_{j=0}^{N} a_{j} L_{j}^{(j)}(t)\right\}+D^{\alpha}\left(\sum_{j=0}^{N} a_{j} L_{j}(t)\right) \\
& +\frac{k}{t^{\alpha-\beta}} D^{\beta}\left(\sum_{j=0}^{N} a_{j} L_{j}(t)\right)+\frac{k}{t^{\alpha-2}}\left(\sum_{j=0}^{N} a_{j} L_{j}(t)\right)=f(t)+H_{n}(t)
\end{aligned}
$$

where

$$
H_{n}(t)=\sum_{(r=1)}^{n} \tau_{r} T_{(N-r-1)}(t)
$$

is called the perturbation term, $n$ is as stated earlier and $T(t)$ is Chebyshev polynomial.

Putting equation (12) into (11), gives

$$
\begin{array}{r}
\sum_{i=0}^{n} P_{i}\left\{\sum_{j=0}^{N} a_{j} L_{j}^{(j)}(t)\right\}+D^{\alpha}\left(\sum_{j=0}^{N} a_{j} L_{j}(t)\right) \\
+\frac{k}{t^{\alpha-\beta}} D^{\beta}\left(\sum_{j=0}^{N} a_{j} L_{j}(t)\right)+\frac{k}{t^{\alpha-2}}\left(\sum_{j=0}^{N} a_{j} L_{j}(t)\right) \\
=f(t)+\sum_{(r=1)}^{n} \tau_{r} T_{(N-r-1)}(t)
\end{array}
$$

subject to conditions

$$
\sum_{k=1}^{n} y_{N}^{(k)}(0)=\phi_{k} ; \quad k=1,2, \ldots n
$$

Where $\tau_{r}(r=1(1) n)$ are the free tau parameters to be determined, $T_{(N-r-1)}(t)$ are the Chebyshev polynomials and $a_{j}$ are the unknown constants also to be determined.

Applying equations (7) on (13) accordingly gives

$$
\sum_{i=0}^{n} P_{i}\left\{\sum_{j=0}^{N} a_{j} L_{j}^{(j)}(t)\right\}+\frac{k}{t^{\alpha-\beta}} \sum_{j=0}^{N} a_{j} \frac{\Gamma(j+1)}{\Gamma(j-\alpha+1)} t^{j-\alpha}
$$

$$
\begin{array}{r}
+\frac{k}{t^{\alpha-2}} \sum_{j=0}^{N} \frac{\Gamma(j+1)}{\Gamma(j-\alpha+1)} t^{j-\alpha}+\sum_{j=0}^{N} a_{j} \frac{\Gamma(j+1)}{\Gamma(j-\beta+1)} t^{j-\beta} \\
=f(t)+\sum_{(r=1)}^{n} \tau_{r} T_{(N-r-1)}(t)
\end{array}
$$

Equation (15) is further simplified and then collocated at equally spaced interior points, $t=t_{e}$ on $[\mathrm{a}, \mathrm{b}] ; t_{e}=a+\frac{(b-a) i}{(N)} ; \quad \mathrm{i}=$ $1,2, \ldots, \mathrm{N}$ to obtain a system of linear algebraic equations, including those obtained from the use of initial conditions. The system of linear algebraic equations are solved using Gaussian elimination method (using a computer package; Maple 18) to obtain the unknown constants. The constants obtained are then substituted back into the assumed approximate solution to give the required approximate solution.

\section{Numerical Examples}

\section{Example 1}

Consider the multi-order fractional differential equation

$$
D^{\alpha}(y(x))+\frac{k}{x^{(\alpha-\beta)}} D^{\beta}(y(x))+\frac{k}{x^{(\alpha-2)}}(y(x))=f(x)
$$

where

$$
\begin{array}{r}
=\left[6 x\left(\frac{\Gamma(4-\beta)+k \Gamma(4-\alpha)}{\Gamma(4-\alpha) \Gamma(4-\beta)}+\frac{x^{2}}{6}\right)\right. \\
\left.-2\left(\frac{\Gamma(3-\beta)+k \Gamma(3-\alpha)}{\Gamma(3-\alpha) \Gamma(3-\beta)}+\frac{x^{2}}{2}\right)\right] x^{2-\alpha}
\end{array}
$$

for $k=1, \alpha=\frac{3}{2}$ and $\beta=\frac{1}{2}$

Subject to the conditions $y(0)=y^{\prime}(0)=0 \quad 0 \leq x \leq 1$

The exact solution is $y(x)=x^{3}-x^{2}$

Equation (16) is perturbed as

$$
\begin{aligned}
D^{\frac{3}{2}}(y(x))+\frac{1}{x^{\left(\frac{3}{2}-\frac{1}{2}\right)}} D^{\frac{1}{2}}(y(x)) & +\frac{1}{x^{\left(\frac{3}{2}-2\right)}}(y(x)) \\
& =f(x)+\sum_{r=1}^{n} \tau_{r} T_{(N-r-1)}(x)
\end{aligned}
$$

Taking an approximate solution for $N=4$

$$
\begin{aligned}
y_{4}=\sum_{j=0}^{4} y(x)= & \sum_{j=0}^{4} a_{j} L_{j}(x)=a_{0}+a_{1}(2 x-1) \\
& +a_{2}\left(6 x^{2}-6 x+1\right) \\
& +a_{3}\left(20 x^{3}-30 x^{2}+12 x-1\right) \\
+ & a_{4}\left(70 x^{4}-140 x^{3}+90 x^{2}-20 x^{2}+1\right)
\end{aligned}
$$

Substituting equations (17) and (19) into (18), we have

$$
D^{\alpha}\left(\sum_{j=0}^{4} y(x)\right)+\frac{1}{x^{(\alpha-\beta)}} D^{\beta}\left(\sum_{j=0}^{4} y(x)\right)+\frac{1}{x^{(\alpha-2)}}\left(\sum_{j=0}^{4} y(x)\right)=
$$




$$
\begin{array}{r}
6 x\left(\frac{\Gamma(4-\beta)+\Gamma(4-\alpha)}{\Gamma(4-\alpha) \Gamma(4-\beta)}+\frac{x^{2}}{6}-2\left(\frac{\Gamma(3-\beta)+\Gamma(3-\alpha)}{\Gamma(3-\alpha) \Gamma(3-\beta)}\right.\right. \\
\left.\left.+\frac{x^{2}}{2}\right)\right] x^{2-\alpha}+\sum_{r=1}^{n} \tau_{r} T_{(N-r-1)}(x)
\end{array}
$$

Further simplifications with $\alpha=\frac{3}{2}$ and $\beta=\frac{1}{2}$ gives

$$
\begin{gathered}
D^{\alpha}\left(a_{0}+a_{1}(2 x-1)+a_{2}\left(6 x^{2}-6 x+1\right)+a_{3}\left(20 x^{3}-30 x^{2}+12 x-1\right)\right. \\
\left.+a_{4}\left(70 x^{4}-140 x^{3}+90 x^{2}-20 x+1\right)\right)+\frac{1}{x} D^{\alpha}\left(a_{0}+a_{1}(2 x-1)\right. \\
+a_{2}\left(6 x^{2}-6 x+1\right)+a_{3}\left(20 x^{3}-30 x^{2}+12 x-1\right)+a_{4}\left(70 x^{4}-140 x^{3}\right. \\
\left.\left.+90 x^{2}-20 x+1\right)\right)+\frac{1}{x^{\frac{-1}{2}}}\left(a_{0}+a_{1}(2 x-1)+a_{2}\left(6 x^{2}-6 x+1\right)\right. \\
\left.+a_{3}\left(20 x^{3}-30 x^{2}+12 x-1\right)+a_{4}\left(70 x^{4}-140 x^{3}+90 x^{2}-20 x+1\right)\right) \\
-\left(6 x\left(\frac{28}{15 \sqrt{\pi}}+1 / 6 x^{2}\right)-\frac{20}{3 \sqrt{\pi}}-x^{2}\right) \sqrt{x} \\
-\tau_{1}\left(8 x^{2}-8 x+1\right)-\tau_{2}(2 x-1)=0
\end{gathered}
$$

Applying the operator $D^{\alpha}$ on equation (21) and after some simplifications gives

$$
-1.7724538509 x^{9 / 2}+1.7724538509 x^{7 / 2}-\frac{56 x^{5 / 2}}{5}+\frac{20 x^{3 / 2}}{3}
$$
$+124.0717696 x^{11 / 2} a_{4}+35.44907702 x^{9 / 2} a_{3}+10.63472311 x^{7 / 2} a_{2}$ $+3.544907702 x^{5 / 2} a_{1}+1.7724538509 x^{3 / 2} a_{0}-3.544907702 \tau_{2} x^{2}$ $-14.17963081 x^{3} \tau_{1}-248.1435391 x^{9 / 2} a_{4}-53.17361553 x^{7 / 2} a_{3}$ $+1311.520847 x^{7 / 2} a_{4}-10.63472311 x^{5 / 2} a_{2}+245.2694462 x^{5 / 2} a_{3}$ $-1603.449077 x^{5 / 2} a_{4}-1.7724538509 x^{3 / 2} a_{1}+41.77245385 x^{3 / 2} a_{2}$ $-201.7724539 x^{3 / 2} a_{3}+601.7724539 x^{3 / 2} a_{4}+6 \sqrt{x} a_{1}-18 \sqrt{x} a_{2}$ $+36 \sqrt{x} a_{3}-60 \sqrt{x} a_{4}+1.7724538509 \tau_{2} x+14.17963081 x^{2} \tau_{1}$

$$
-1.7724538509 x \tau_{1}=0
$$

Equation (22) is collocated at $N-2$ equal spaced points in the interval $[0,1]$ and the remaining two equations are obtained from the use of the conditions attached. This gives 7 system of linear equations. Solving the system of equations, we get

$$
\begin{array}{r}
y_{4}(x)=-1.48141262 \times 10^{-10}+3.2 \times 10^{-9} x-1.000000039 x^{2} \\
+1.00000004 x^{3}-5.469888349 \times 10^{-9} x^{4}
\end{array}
$$

When the same example was solved $\alpha=\frac{5}{2}, \beta=\frac{3}{2}$ following the same methodology,the approximate solution obtained is

$$
\begin{array}{r}
y_{4}(x)=5.00 \times 10^{-11}-3 \times 10^{-10} x-1.397902893 x^{2} \\
+1.664907851 x^{3}-0.1566880882 x^{4}
\end{array}
$$

\section{Example 2}

Consider the multi-order fractional differential equation

$$
D^{\alpha}(y(x))+\frac{k}{x^{(\alpha-\beta)}} D^{\beta}(y(x))+\frac{k}{x^{(\alpha-2)}}(y(x))=
$$

$$
\begin{aligned}
& {\left[2\left(\frac{\Gamma(3-\beta)+k \Gamma(3-\alpha)}{\Gamma(3-\alpha) \Gamma(3-\beta)}+\frac{x^{2}}{2}\right)-6 x\left(\frac{\Gamma(4-\beta)+k \Gamma(4-\alpha)}{\Gamma(4-\alpha) \Gamma(4-\beta)}\right.\right.} \\
& \left.\left.+\frac{x^{2}}{6}\right)\right] x^{2-\alpha}
\end{aligned}
$$

for $k=1, \alpha=\frac{3}{2}$ and $\beta=\frac{1}{2}$

subject to conditions $y(0)=y^{\prime}(0)=0 \quad 0<x \leq 1$ with exact solution $y(x)=-x^{3}+x^{2}$

Taking an assumed approximate solution for $N=4$, and computing the $\alpha=\frac{3}{2}$ and $\beta=\frac{1}{2}$-derivatives of equation (25) and other necessary simplifications and substitution, gives

$35.44907702 x^{9 / 2} a_{3}+10.63472311 x^{7 / 2} a_{2}+3.544907702 x^{5 / 2} a_{1}$ $+1.7724538509 x^{3 / 2} a_{0}-3.544907702 \tau_{2} x^{2}-14.17963081 x^{3} \tau_{1}$ $-248.1435391 x^{9 / 2} a_{4}-53.17361553 x^{7 / 2} a_{3}+1311.520847 x^{7 / 2} a_{4}$ $-10.63472311 x^{5 / 2} a_{2}+245.2694462 x^{5 / 2} a_{3}-1603.449077 x^{5 / 2} a_{4}$ $-1.7724538509 x^{3 / 2} a_{1}+41.77245385 x^{3 / 2} a_{2}-201.7721531 x^{3 / 2} a_{3}$ $+1.7724538509 x^{9 / 2}+124.0717696 x^{11 / 2} a_{4}+601.7724539 x^{3 / 2} a_{4}$ $+6 \sqrt{x} a_{1}-18 \sqrt{x} a_{2}+36 \sqrt{x} a_{3}-60 \sqrt{x} a_{4}+1.7724538509 \tau_{2} x$ $+14.17963081 x^{2} \tau_{1}-1.7724538509 x \tau_{1}-1.7724538509 x^{7 / 2}$

$$
-20 x^{3 / 2}+51 x^{2 / 3}=0
$$

Equation (26) is collocated at $N-2$ equal spaced points in the interval $[0,1]$ and the remaining two equations are obtained from the use of the conditions attached. This gives 7 system of linear equations. Solving the system of equations, we get

$$
\begin{gathered}
y_{4}(x)=3.77824 \times 10^{-12}-1 . \times 10^{-10} x+1.000000109 x^{2} \\
-0.9999984717 x^{3}-0.1415135523 e-5 x^{4}
\end{gathered}
$$

When the same example was solved $\alpha=\frac{5}{2}, \beta=\frac{3}{2}$ following the same method,the approximate solution obtained is

$$
\begin{array}{r}
y_{4}(x)=6.2 \times 10^{-9}-1.449037318 x^{2}-8.450067824 x^{3} \\
+5.265645764 x^{4}
\end{array}
$$

\section{Example 3}

Consider the multi-order fractional differential equation

$$
\begin{aligned}
D^{\alpha}(y(x))+\frac{2}{x} D^{\beta}(y(x))+ & x y(x)=x^{2}+x^{3}+x^{4} \\
& +\frac{96 \sqrt{x}}{\sqrt{\pi}}+\frac{3}{\sqrt{\pi} \sqrt{x}}\left(6+\frac{1}{\sqrt{x}}\right)
\end{aligned}
$$

subject to conditions $y(0)=y^{\prime}(0)=0 \quad 0<x \leq 1$ with exact solution $y(x)=x+x^{2}+x^{3}$

Taking an assumed approximate solution for $N=4$, and computing the $\alpha=\frac{5}{2}$ and $\beta=\frac{3}{2}$-derivatives of equation (29) and other necessary simplifications and substitution, gives

$$
70 x^{5} a_{4}+20 x^{4} a_{3}-140 x^{4} a_{4}+6 x^{3} a_{2}-30 x^{3} a_{3}+1490 x^{3} a_{4}
$$$$
+2 x^{2} a_{1}-6 x^{2} a_{2}+252 x^{2} a_{3}-1700 x^{2} a_{4}+x a_{0}-x a_{1}+37 x a_{2}
$$ 
$-181 x a_{3}+541 x a_{4}+4 a_{1}-12 a_{2}+24 a_{3}-40 a_{4}=x^{4}+8 x^{3} \tau_{1}+x^{3}$

$$
-8 x^{2} \tau_{1}+2 x^{2} \tau_{2}+12 x^{2}+x \tau_{1}-x \tau_{2}+x \tau_{3}+x
$$

Equation (30) is collocated at $N-2$ equal spaced points in the interval $[0,1]$ and the remaining two equations are obtained from the use of the conditions attached. This gives 8 system of linear equations. Solving the system of equations, we get

$$
\begin{array}{r}
y_{4}(x)=3.2749743 \times 10^{(-10)}+1.6 \times 10^{(-8)} x+1.109848402 x^{2} \\
+1.000005386 x^{3}-0.2049775180 e-5 x^{4}
\end{array}
$$

When the same example was solved $\alpha=\frac{3}{2}, \beta=\frac{1}{2}$ following the same method,the approximate solution obtained is

$$
\begin{array}{r}
y_{4}(x)=-4.9638971 \times 10^{(-10)}+2.6 \times 10^{(-8)} x+1.48521236 x^{2} \\
+1.000023102 x^{3}-0.346404728 e-5 x^{4}
\end{array}
$$

\section{Example 4}

Consider the multi-order fractional differential equation

$$
D^{\alpha}(y(x))+\frac{8}{x} D^{\beta}(y(x))+x y(x)=x^{5}-x^{4}+44 x^{2}-30 x(33)
$$

subject to conditions $y(0)=y^{\prime}(0)=0 \quad 0<x \leq 1$

with exact solution $y(x)=x^{4}-x^{3}$

Taking an assumed approximate solution for $N=4$, and computing the $\alpha=\frac{5}{2}$ and $\beta=\frac{3}{2}$-derivatives of equation (33) and other necessary simplifications and substitution, gives

$$
\begin{gathered}
-140 x^{5} a_{4}+6 x^{4} a_{2}-30 x^{4} a_{3}+370 x^{4} a_{4}+2 x^{3} a_{1}-6 x^{3} a_{2} \\
+72 x^{3} a_{3}+1800 x^{3} a_{4}+x^{2} a_{0}-x^{2} a_{1}+13 x^{2} a_{2}+419 x^{2} a_{3} \\
-3179 x^{2} a_{4}+2 x a_{1}+9 x a_{2}+70 x^{6} a_{4}+20 x^{5} a_{3}-468 x a_{3} \\
+142 x a_{4}+16 a_{1}-48 a_{2}+96 a_{3}-160 a_{4} \\
=x^{6}-x^{5}+8 x^{3} \tau_{1}+44 x^{3}-8 x^{2} \tau_{1}+2 x^{2} \tau_{2}-30 x^{2}+x \tau_{1}-x \tau_{2}
\end{gathered}
$$

Equation (34) is collocated at $N-2$ equal spaced points in the interval $[0,1]$ and the remaining two equations are obtained from the use of the conditions attached. This gives 8 system of linear equations. Solving the system of equations, we obtained

$$
\begin{array}{r}
y_{4}(x)=-8 . \times 10^{(-11)}+1 . \times 10^{(-9)} x-2.857225303 x^{2} \\
+4.713873487 x^{3}-.9046244956 x^{4}
\end{array}
$$

When the same example was solved $\alpha=\frac{3}{2}, \beta=\frac{1}{2}$ following the same method,the approximate solution obtained is

$$
y_{4}(x)=-1 . \times 10^{(-10)} x-1.000000001 x^{3}+1.000000000 x^{4}(36)
$$

\section{Conclusion}

In this study, the proposed method was used to solve multiorder fractional differential equations successfully. Four examples were solved and the results of the numerical solutions presented in Tables 1, 2, 3 and 4. When $\alpha=\frac{3}{2}$ and $\beta=\frac{1}{2}$ the proposed method produced accurate approximate solution curve

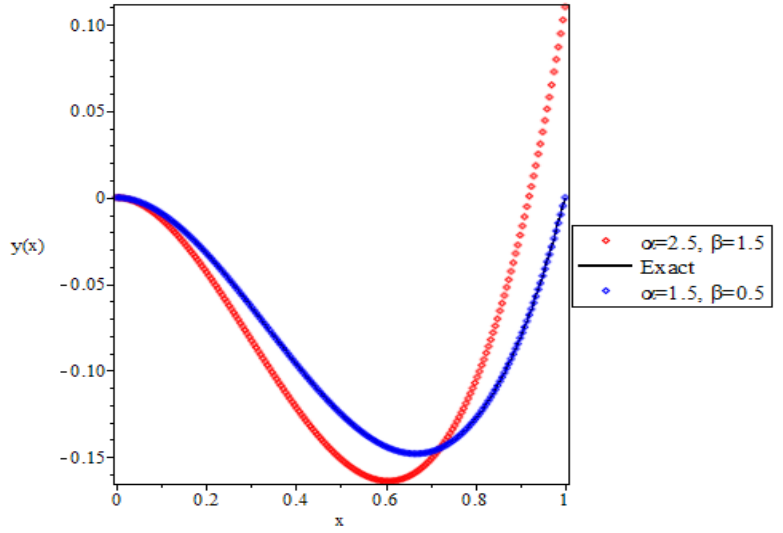

Figure 1. Graphical Representation of Error in Table 1

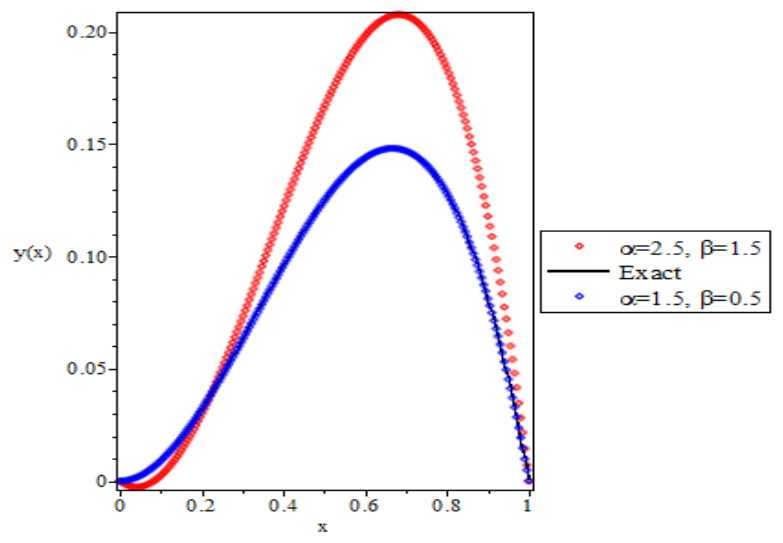

Figure 2. Graphical Representation of Error in Table 2

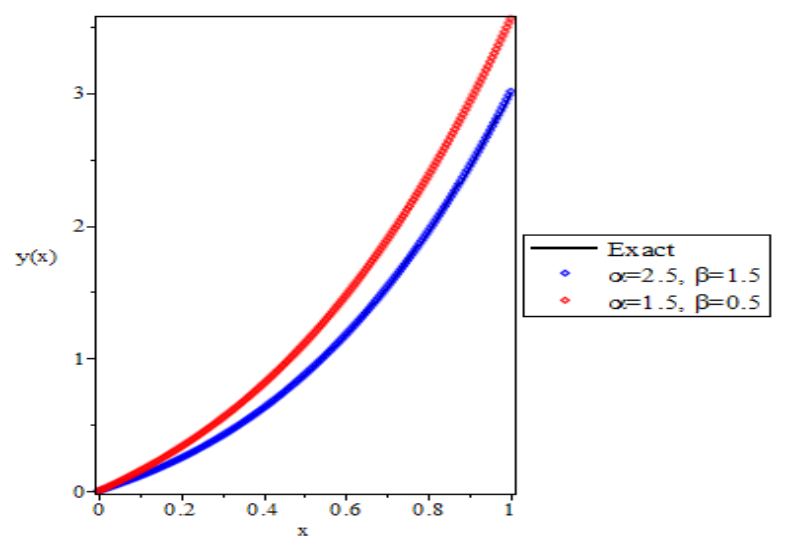

Figure 3. Graphical Representation of Error in Table 3

which is exactly over the curve of the exact solution for examples 1, and 2. But, the results are not as satisfactory at $\alpha=\frac{5}{2}$ and $\beta=\frac{3}{2}$ as seen in Figures 1 and 2. However, for examples 3 and 4 , there is a change of results. Here, the results are better at $\alpha=\frac{5}{2}$ and $\beta=\frac{3}{2}$ than at $\alpha=\frac{3}{2}$ and $\beta=\frac{1}{2}$ as shown in Figure 3 and 4 . This is likely because $k$ in these examples is higher than 1 which is 2 and 8 in examples 3 and 4 respectively. The value 
Table 1. Error of Results for Example 1

\begin{tabular}{|c|c|c|c|c|c|}
\hline $\mathrm{x}$ & & $\alpha=\frac{5}{2}$ & & $\alpha=\frac{3}{2}$ & \\
\hline & & $\beta=\frac{3}{2}$ & & $\beta=\frac{1}{2}$ & \\
\hline & Exact & Appx & Error & Appx & Error \\
\hline 0.0 & 0.00000 & 0.00000 & $5.0000 \mathrm{e}-11$ & -0.000000 & $5.9232 \mathrm{e}-13$ \\
\hline 0.1 & 0.00900 & -0.01232 & $3.3298 \mathrm{e}-03$ & 0.008999 & $2.6668 \mathrm{e}-10$ \\
\hline 0.2 & 0.03200 & -0.04284 & $1.0848 \mathrm{e}-02$ & 0.031999 & $9.6994 \mathrm{e}-10$ \\
\hline 0.3 & 0.06300 & -0.08213 & $1.9128 \mathrm{e}-02$ & 0.062999 & $1.9604 \mathrm{e}-09$ \\
\hline 0.4 & 0.09600 & -0.12112 & $2.5122 \mathrm{e}-02$ & 0.095999 & $3.0781 \mathrm{e}-09$ \\
\hline 0.5 & 0.12500 & -0.15115 & $2.6155 \mathrm{e}-02$ & 0.124999 & $4.1532 \mathrm{e}-09$ \\
\hline 0.6 & 0.14400 & -0.16393 & $1.9932 \mathrm{e}-02$ & 0.144000 & $5.0056 \mathrm{e}-09$ \\
\hline 0.7 & 0.14700 & -0.15153 & $4.5298 \mathrm{e}-03$ & 0.146999 & $5.4456 \mathrm{e}-09$ \\
\hline 0.8 & 0.12800 & -0.10640 & $2.1596 \mathrm{e}-02$ & 0.127999 & $5.2733 \mathrm{e}-09$ \\
\hline 0.9 & 0.08100 & -0.02139 & $5.9613 \mathrm{e}-02$ & 0.080999 & $4.2787 \mathrm{e}-09$ \\
\hline 1.0 & 0.00000 & 0.11032 & $1.1032 \mathrm{e}-01$ & 0.000000 & $2.2421 \mathrm{e}-09$ \\
\hline
\end{tabular}

Table 2. Error of Results for Example 2

\begin{tabular}{|c|c|c|c|c|c|}
\hline $\mathrm{x}$ & & $\alpha=\frac{5}{2}$ & & $\alpha=\frac{3}{2}$ & \\
\hline & & $\beta=\frac{3}{2}$ & & $\beta=\frac{1}{2}$ & \\
\hline & Exact & Appx & Error & Appx & Error \\
\hline 0.0 & 0.000000 & 0.000000 & $6.2000 \mathrm{e}-10$ & 0.000000 & $3.7782 \mathrm{e}-12$ \\
\hline 0.1 & 0.009000 & -0.022414 & $3.1414 \mathrm{e}-02$ & 0.0090000 & $2.4706 \mathrm{e}-09$ \\
\hline 0.2 & 0.032000 & -0.117137 & $1.4914 \mathrm{e}-01$ & 0.0320000 & $1.4306 \mathrm{e}-08$ \\
\hline 0.3 & 0.063000 & -0.315914 & $3.7891 \mathrm{e}-01$ & 0.0630000 & $3.9585 \mathrm{e}-08$ \\
\hline 0.4 & 0.096000 & -0.637850 & $7.3385 \mathrm{e}-01$ & 0.0960000 & $7.8988 \mathrm{e}-08$ \\
\hline 0.5 & 0.125000 & -1.089415 & $1.2144 \mathrm{e}+00$ & 0.125000 & $1.2980 \mathrm{e}-07$ \\
\hline 0.6 & 0.144000 & -1.664440 & $1.8084 \mathrm{e}+00$ & 0.1440001 & $1.8590 \mathrm{e}-07$ \\
\hline 0.7 & 0.147000 & -2.344120 & $2.4911 \mathrm{e}+00$ & 0.1470002 & $2.3778 \mathrm{e}-07$ \\
\hline 0.8 & 0.128000 & -3.097010 & $3.2250 \mathrm{e}+00$ & 0.1280002 & $2.7253 \mathrm{e}-07$ \\
\hline 0.9 & 0.081000 & -3.879029 & $3.9600 \mathrm{e}+00$ & 0.0810002 & $2.7386 \mathrm{e}-07$ \\
\hline 1.0 & 0.000000 & -4.633459 & $4.6335 \mathrm{e}+00$ & 0.0000002 & $2.2207 \mathrm{e}-07$ \\
\hline
\end{tabular}

Table 3. Error of Results for Example 3

\begin{tabular}{|c|c|c|c|c|c|}
\hline $\mathrm{x}$ & & $\alpha=\frac{3}{2}$ & & $\alpha=\frac{5}{2}$ & \\
\hline & & $\beta=\frac{1}{2}$ & & $\beta=\frac{3}{2}$ & \\
\hline & Exact & Appx & Error & Appx & Error \\
\hline 0.0 & 0.0000000000 & -0.0000000005 & $4.9639 \mathrm{e}-10$ & 0.0000000000 & $3.4707 \mathrm{e}-11$ \\
\hline 0.1 & 0.0110000000 & 0.0158521485 & $4.8521 \mathrm{e}-03$ & 0.0110000001 & $8.6920 \mathrm{e}-11$ \\
\hline 0.2 & 0.0480000000 & 0.0674086784 & $1.9409 \mathrm{e}-02$ & 0.0480000002 & $2.1491 \mathrm{e}-10$ \\
\hline 0.3 & 0.1170000000 & 0.1606697155 & $4.3670 \mathrm{e}-02$ & 0.1170000004 & $3.8136 \mathrm{e}-10$ \\
\hline 0.4 & 0.2240000000 & 0.3016353775 & $7.7635 \mathrm{e}-02$ & 0.2240000006 & $5.5642 \mathrm{e}-10$ \\
\hline 0.5 & 0.3750000000 & 0.4963057740 & $1.2131 \mathrm{e}-01$ & 0.3750000007 & $7.1780 \mathrm{e}-10$ \\
\hline 0.6 & 0.5760000000 & 0.7506810062 & $1.7468 \mathrm{e}-01$ & 0.5760000009 & $8.5069 \mathrm{e}-10$ \\
\hline 0.7 & 0.8330000000 & 1.0707611670 & $2.3776 \mathrm{e}-01$ & 0.8330000009 & $9.4779 \mathrm{e}-10$ \\
\hline 0.8 & 1.1520000000 & 1.4625463410 & $3.1055 \mathrm{e}-01$ & 1.1520000010 & $1.0093 \mathrm{e}-09$ \\
\hline 0.9 & 1.5390000000 & 1.9320366030 & $3.9304 \mathrm{e}-01$ & 1.5390000010 & $1.0431 \mathrm{e}-09$ \\
\hline 1.0 & 2.0000000000 & 2.4852320250 & $4.8523 \mathrm{e}-01$ & 2.0000000010 & $1.0642 \mathrm{e}-09$ \\
\hline
\end{tabular}


Table 4. Error of Results for Example 4

\begin{tabular}{|c|c|c|c|c|c|}
\hline $\mathrm{x}$ & & $\alpha=\frac{3}{2}$ & & $\alpha=\frac{5}{2}$ & \\
\hline & & $\beta=\frac{1}{2}$ & & $\beta=\frac{3}{2}$ & \\
\hline & Exact & Appx & Error & Appx & Error \\
\hline 0.0 & 0.0000000000 & -0.0000000001 & $8.0000 \mathrm{e}-11$ & 0.0000000000 & $0.0000 \mathrm{e}+00$ \\
\hline 0.1 & -0.0009000000 & -0.0239488420 & $2.3049 \mathrm{e}-02$ & -0.0009000000 & $1.1000 \mathrm{e}-11$ \\
\hline 0.2 & -0.0064000000 & -0.0780254233 & $7.1625 \mathrm{e}-02$ & -0.0064000000 & $2.8000 \mathrm{e}-11$ \\
\hline 0.3 & -0.0189000000 & -0.1372031514 & $1.1830 \mathrm{e}-01$ & -0.0189000001 & $5.7000 \mathrm{e}-11$ \\
\hline 0.4 & -0.0384000000 & -0.1786265321 & $1.4023 \mathrm{e}-01$ & -0.0384000001 & $1.0400 \mathrm{e}-10$ \\
\hline 0.5 & -0.0625000000 & -0.1816111705 & $1.1911 \mathrm{e}-01$ & -0.0625000002 & $1.7500 \mathrm{e}-10$ \\
\hline 0.6 & -0.0864000000 & -0.1276437696 & $4.1244 \mathrm{e}-02$ & -0.0864000003 & $2.7600 \mathrm{e}-10$ \\
\hline 0.7 & -0.1029000000 & -0.0003821324 & $1.0252 \mathrm{e}-01$ & -0.1029000004 & $4.1300 \mathrm{e}-10$ \\
\hline 0.8 & -0.1024000000 & 0.2143448386 & $3.1674 \mathrm{e}-01$ & -0.1024000006 & $5.9200 \mathrm{e}-10$ \\
\hline 0.9 & -0.0729000000 & 0.5285371464 & $6.0144 \mathrm{e}-01$ & -0.0729000008 & $8.1900 \mathrm{e}-10$ \\
\hline 1.0 & 0.0000000000 & 0.9520236894 & $9.5202 \mathrm{e}-01$ & -0.0000000010 & $1.1000 \mathrm{e}-09$ \\
\hline
\end{tabular}

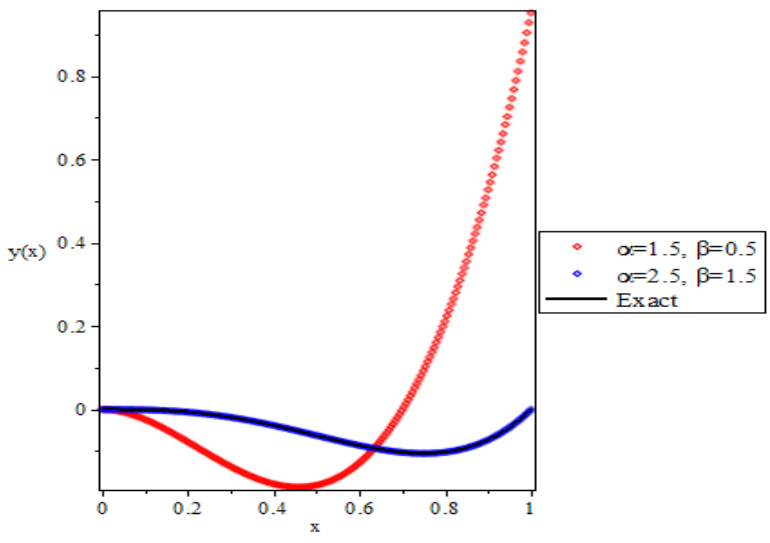

Figure 4. Graphical Representation of Error in Table 4

of the exact solution and the source functions count. From numerical results, it can be seen that the proposed method is an accurate estimate for the class of differential equations considered.

\section{Acknowledgments}

The authors wish to sincerely appreciate Mr Akorede M. T, for his immense contributions in the typesetting of this work. We are also grateful to the referees and editor for their invaluable comments and suggestions.

\section{References}

[1] S. Abbasbandy. "Application of He's homotopy perturbation method for Laplace transform", Chaos Solution \& Fractals 30 (2006) 1206.

[2] M. Dehghan \& F. Shakeri. "Solution of an integro-differential equation arising in Oscillating Magnetic Fields using He's Homotopy Perturbation Method", PIER 78 (2008) 361.
[3] Z. Odibat \& S. Momani. "Modified homotopy perturbation method: application to quadratic riccati differential equation of fractional order", Chaos, Solution and Fractals 36 (2008) 167.

[4] I. K. Omar. "Variational iteration method for solving multi fractional integrodifferential equations", Iraqi Journal of Science 55 (2014) 1086.

[5] A. Yildirim \& T. Qzis. "Solution of singular IVPs of Lane-Emden type by variational iteration method", Nonlinear Analysis: Theory, Method \& Applications 70 (2009) 2480. doi.org/10.1016/j.na.2008.03.012

[5] Y. Yang. "Solving a nonlinear multi-order fractional differential equation using Legendre pseudo-spectral method", Journal of Applied Mathematics 4 (2013) 113.

[6] L. Jianping, L. Xia, X. Liyong \& S. Yufa. "Solution of fractional differential equations by Bernstein polynomials method", Journal of Convergence Information Technolgy 7 (2012) 820.

[7] A. Barzkar, M. Oshagh, P. Assari \& M. Mehrpouya. "Numerical solution of the nonlinear Fredholm integral equation and Fredholm integro-differential equation of second kind using Chebyshev wavelets", World Applied Sciences Journal 18 (2012) 1774. doi.org/10.5829/idosi.wasj.2012.18.12.920.

[8] Z. Avazzadeh \& M. Heydari. "Chebyshev polynomials for solving two dimensional linear and nonlinear integral equations of the second kind". Computational and Applied Mathematics 31 (2012) 127.

[9] H. Cerdik-Yaslan \& Z. Akyuz-Dascioglu. "Chebyshev polynomial solution of nonlinear Fredholm-Volterra integro-differential equation", Journal of Arts Science 5 (2006) 89.

[10] O. A. Uwaheren \& O. A. Taiwo. "A collocation technique based on an orthogonal polynomial for solving multi-fractional order integro differential equations", Journal Mathematical Society of Nigeria (ABACUS) $\mathbf{4 3}$ (2016) 215.

[11] M. Yigider. "The numerical method for solving differential equations of Lane-Emden type by Pade approximation", Discrete Dynamics in Nature and Society (2011) 1. doi.org/10.1155/2011/479396

[12] S. M. Mohammed \& S. Norazak. "Numerical study of fractional differential equations of Lane-Emden type by method of collocation", Applied Mathematics 3 (2012) 851. doi.org/10.4236/am.2012.38126.

[13] A. Verma \& M. Kumar. "Numerical solution of Lane-Emden type equation using multilayer perceptron neural network method", International Journal of Applied and Computational Mathematics 5 (2019) 141. link.springer.com

[14] A. H. Bhrawy \& S. A. Alofi. "A Jacob Gauss collocation method for solving nonlinear Lane-Emden type equation", Communications in Nonlinear Science and Numerical Simulation 17 (201) 62.

[15] K. Parand, M. Dehgham, A. Rezaeia \& S. Ghaderi. "An applica- 
tion algorithm for the solution of the nonlinear Lane-Emden type equations arising in astrophysics using Hermite functions collocation method", Computer Physics Communications 181 (2010) 1096. doi.org/10.1016/j.cpc.2010.02.018
[16] W. Al-Hayani, L. Alzubaidy \& A. Entesar. "Solutions of singular IVPs of Lane-Emden type by homotopy analysis method with genetic algorithm”, Applied Mathematics and Information Sciences 11 (2019) 1. doi.org/10.18576/amis/paper 